\title{
Numerical Solution of the Dirichlet Problem for Systems of Circular Conductors between Parallel Ground Lines
}

\author{
By David W. Kammler
}

Abstract. A Green's function-integral equation technique is used to obtain a numerical solution to the two-dimensional Dirichlet problem for the multiply connected region between the ground lines $(V=0)$ at $y=0$ and $y=1$ and exterior to the $N$ circular conductors with arbitrarily spaced centers $\left(x_{i}, y_{i}\right)$ and radii $R_{i}, i=$ $1,2, \cdots, N$. If the smallest distance between each pair of conducting surfaces exceeds .1 , the capacitance matrix for this system of $N$ conductors can be calculated to 6-place accuracy at a cost of about $15 N^{2}$ seconds on the IBM 7044 computer.

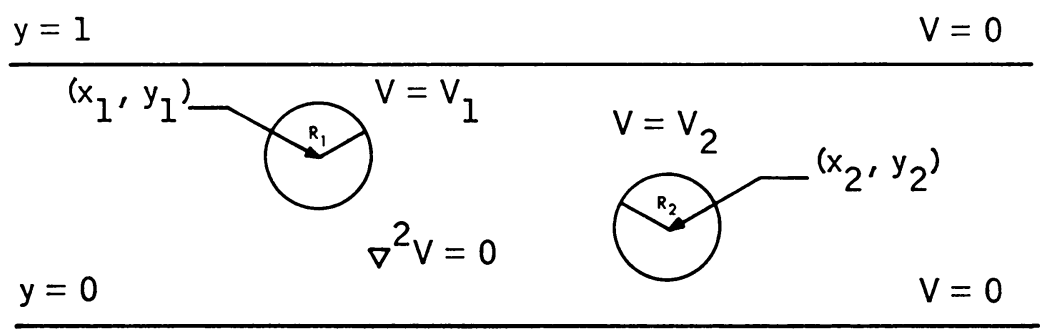

Figure 1. A System of $N=2$ Circular Conductors

1. The Problem. A two-dimensional system of nonintersecting circular conductors is located in the strip between $y=0$ and $y=1$ with the $i$ th conductor having radius $R_{i}$ and center $\left(x_{i}, y_{i}\right), i=1,2, \cdots, N$. It is supposed that none of the conductors intersects the ground lines at $y=0$ and $y=1$. We wish to find a function $V(x, y)$ such that

$$
\begin{aligned}
\nabla^{2} V(x, y)=0 \text { for }\left\{\begin{array}{l}
-\infty<x<\infty, \\
0<y<1, \\
\left(x-x_{i}\right)^{2}+\left(y-y_{i}\right)^{2}>R_{i}{ }^{2}, i=1,2, \cdots, N
\end{array}\right. \\
V(x, y)=0 \text { for } y=0 \text { and } y=1, \\
V(x, y) \rightarrow 0 \text { as } x \rightarrow \pm \infty, \\
V(x, y)=V_{i} \text { for }\left(x-x_{i}\right)^{2}+\left(y-y_{i}\right)^{2} \leqq{R_{i}}_{i}{ }^{2} i=1,2, \cdots, N .
\end{aligned}
$$

Problems of a similar nature have been considered by Knight [1], Craggs [2], and Cristal [3]. We would also like to calculate the elements of the capacitance matrix $\left\{C_{i j}\right\}$ (which is independent of the boundary conditions of Eq. (3)) such that if

$$
Q_{i}=-\epsilon \oint_{i \mathrm{th}} \frac{\partial V}{\partial n} d l
$$

Received November 9, 1967, revised July 22, 1968. 
is the charge (in $M K S$ units) on the $i$ th conductor, then

$$
Q_{i}=\sum_{j=1}^{N} C_{i j} V_{j}
$$

(The constant $\epsilon$ is the electrical permittivity constant.) We may obtain the $j$ th column of this matrix by noting that $Q_{i}=C_{i j}$ for the special case where the boundary conditions of Eq. (3) are chosen such that $V_{i}=\delta_{i j}$ where $\delta_{i j}$ is the Dirac delta function.

2. The Corresponding Integral Equation. In the usual manner we may convert the above Dirichlet problem to an integral equation [4]. Accordingly let $\sigma_{i}(\theta)$ be the angular charge density, i.e. $-\epsilon R_{i}$ times the outward normal derivative of $V(x, y)$, at the angular coordinate $\theta$ on the surface of the $i$ th conductor. (Here $\theta$ is measured counterclockwise from the ray drawn from $\left(x_{i}, y_{i}\right)$ to $\left(+\infty, y_{i}\right)$.) Then we have

$$
V(x, y)=\sum_{i=1}^{N} \int_{\theta=0}^{2 \pi} G\left(x, y ; x_{i}+R_{i} \cos \theta, y_{i}+R_{i} \sin \theta\right) \sigma_{i}(\theta) d \theta
$$

where

$$
G\left(x, y ; x^{\prime}, y^{\prime}\right)=-\frac{1}{4 \pi \epsilon} \log \frac{\cosh \pi\left(x-x^{\prime}\right)-\cos \pi\left(y-y^{\prime}\right)}{\cosh \pi\left(x-x^{\prime}\right)-\cos \pi\left(y+y^{\prime}\right)}
$$

is the Green's function for the region between the ground lines [5], i.e. $G\left(x, y ; x^{\prime}, y^{\prime}\right)$ is the potential at the point $(x, y)$ due to a unit charge at the point $\left(x^{\prime}, y^{\prime}\right)$ in the presence of the ground lines at $y=0, y=1$. By construction the $V(x, y)$ of Eq. (5) satisfies Eq. (1) and the boundary conditions of Eq. (2) for any choice of the functions $\sigma_{i}(\theta)$; we shall select the latter in such a way as to insure that the boundary conditions of Eq. (3) are also satisfied. The boundary conditions of Eq. (3) and the integral relation of Eq. (5) imply that the functions $\sigma_{i}(\theta)$ have continuous derivatives of all orders, and consequently we expect the Fourier series

$$
\sigma_{i}(\theta)=a_{i 0}+\sum_{m=1}^{\infty}\left[a_{i m} \cos m \theta+b_{i m} \sin m \theta\right]
$$

to converge rapidly. After substituting Eq. (7) into Eq. (5) and interchanging the summation and integration processes, we obtain

$$
\begin{aligned}
V(x, y)=\sum_{i=1}^{N}\left\{a_{i 0} A_{0}\left(x, y ; x_{i}, y_{i}\right)\right. & \\
& \left.\quad+\sum_{m=1}^{\infty} R_{i}{ }^{m}\left[a_{i m} A_{m}\left(x, y ; x_{i}, y_{i}\right)+b_{i m} B_{m}\left(x, y ; x_{i}, y_{i}\right)\right]\right\}
\end{aligned}
$$

where

$$
\begin{aligned}
& A_{m}\left(x, y ; x_{0}, y_{0}\right) \equiv R^{-m} \int_{0}^{2 \pi} G\left(x, y ; x_{0}+R \cos \theta, y_{0}+R \sin \theta\right) \cos m \theta d \theta, \\
& B_{m}\left(x, y ; x_{0}, y_{0}\right) \equiv R^{-m} \int_{0}^{2 \pi} G\left(x, y ; x_{0}+R \cos \theta, y_{0}+R \sin \theta\right) \sin m \theta d \theta .
\end{aligned}
$$

(It will be seen later that the functions $A_{m}, B_{m}$ as defined here are independent of $R$ for $R>0$.) It will be noted that each of the functions $A_{m}\left(x, y ; x_{0}, y_{0}\right), m=0,1$, 
$2, \cdots$ and $B_{m}\left(x, y ; x_{0}, y_{0}\right), m=1,2,3, \cdots$ satisfies the boundary conditions of Eq. (2), and each satisfies Laplace's equation at any point $(x, y)$ with $0<y<1$ and $(x, y) \neq\left(x_{0}, y_{0}\right)$.

3. Numerical Determination of $a_{i m}, b_{i m}$. Upon truncating the infinite series of Eq. (8) we obtain the $M$ th order approximation

$$
\begin{aligned}
V^{(M)}(x, y)=\sum_{i=1}^{N}\left\{a_{i 0}^{(M)} A_{0}\left(x, y ; x_{i}, y_{i}\right)\right. & \\
& \left.+\sum_{m=1}^{M} R_{i}{ }^{m}\left[a_{i m}^{(M)} A_{m}\left(x, y ; x_{i}, y_{i}\right)+b_{i m}^{(M)}\left(x, y ; x_{i}, y_{i}\right)\right]\right\}
\end{aligned}
$$

so that $V^{(M)}$ is a finite linear combination of the functions $A_{m}, B_{m}$ and therefore satisfies the boundary conditions of Eq. (2) and satisfies Laplace's equation everywhere in the strip $0<y<1$ except at the points $\left(x_{i}, y_{i}\right), i=1,2, \cdots, N$. We shall force $V^{(M)}$ to satisfy approximately the remaining boundary conditions of Eq. (3) by choosing the Fourier coefficients $a_{i m}^{(M)}, b_{i m}^{(M)}$ such that

$$
\sum_{i=1}^{N} \sum_{k=1}^{4 M}\left\{V^{(M)}\left(X_{i k}, Y_{i k}\right)-V_{i}\right\}^{2}=\text { Minimum , }
$$

where

$$
\begin{aligned}
& X_{i k}=x_{i}+R_{i} \cos (k \pi / 2 M), \\
& Y_{i k}=y_{i}+R_{i} \sin (k \pi / 2 M),
\end{aligned} \quad i=1,2, \cdots, N ; k=1,2, \cdots, 4 M,
$$

is a set of equally spaced test points chosen on the surfaces of the $N$ conductors. The least squares condition of Eq. (11) gives rise to a system of $N \cdot(2 M+1)$ linear equations in the $N \cdot(2 M+1)$ unknowns $a_{i m}^{(M)}, b_{i m}^{(M)}$ which are thereby determined. A total of $4 N M$ test points is specified by Eq. (12), since the use of approximately twice as many test points as unknown Fourier coefficients makes a near optimum use of the smoothing effect of the least squares process.

Numerically this computational procedure has been found to be very stable, and as $M \rightarrow \infty$ the $M$ th order potential function $V^{(M)}$ rapidly converges to the solution of the Dirichlet problem of Eqs. (1)-(3). Likewise, the $M$ th order charge densities $\sigma_{i}{ }^{(M)}$ converge to their limiting forms $\sigma_{i}$, thus satisfying the integral equation associated with the Dirichlet problem. The $M$ th order estimate of the charge on the $i$ th conductor is given by the integral of $\sigma_{i}{ }^{(M)}$, i.e.

$$
Q_{i}^{(M)}=\int_{0}^{2 \pi}\left\{a_{i 0}^{(M)}+\sum_{m=1}^{M}\left[a_{i m}^{(M)} \cos m \theta+b_{i m}^{(M)} \sin m \theta\right]\right\} d \theta=2 \pi a_{i 0}^{(M)}
$$

and by using the boundary conditions $V_{i}=\delta_{i j}$ we may thereby obtain an $M$ th order estimate of the $(i, j)$-element of the capacitance matrix of Eq. (4).

The extent to which the approximate potential function $V^{(M)}$ satisfies the boundary condition of Eq. (3) on the selected test points $\left(X_{i k}, Y_{i k}\right)$ gives a good indication of the degree of convergence attained for a particular value of $M$. Another useful test of convergence may be used when the complete capacitance matrix of Eq. (4) is to be determined for a system of $N \geqq 2$ conductors. The exact matrix is symmetric [6], and the extent to which $C_{i j}^{(M)}$ agrees with $C_{j i}^{(M)}, i \neq j=1,2, \cdots, N$ gives a good indication of the accuracy of the $M$ th order estimates.

To give some idea of the convergence, it will be noted that if the smallest distance 
between any pair of conducting surfaces (ground lines or circular conductors) exceeds about .1, then the use of $M=6$ will give approximations to $V(x, y), \sigma_{i}(\theta)$, and $Q_{i}, i=1,2, \cdots, N$ which are all accurate to about 6 decimal places. When one of the circular conductors is closer than about 11 to one of the ground planes or to another circular conductor having a different boundary condition, then the corresponding exact charge density $\sigma_{i}(\theta)$ will be quite peaked and therefore a greater number of terms of the Fourier series will be needed to obtain a 6-place approximation to $\sigma_{i}(\theta)$. This in turn requires the use of a correspondingly larger $M$ to obtain 6-place approximations to $V(x, y)$ and the charges $Q_{i}, i=1,2, \cdots, N$.

4. Numerical Evaluation of $A_{m}, B_{m}$. The preceding analysis requires the determination of each of the functions $A_{m}, B_{m}, m=0,1,2, \cdots, M$, at each of the $4 N^{2} M$ arguments $\left(X_{i k}, Y_{i k} ; x_{j}, y_{j}\right), i, j=1,2, \cdots, N ; k=1,2, \cdots, 4 M$ in order to determine the $N(2 M+1)$ Fourier coefficients $a_{i m}^{(M)}, b_{i m}^{(M)}$. In addition these functions must be evaluated at the $N$ arguments $\left(x, y ; x_{i}, y_{i}\right), i=1,2, \cdots, N$ whenever the potential $V^{(M)}(x, y)$ is calculated. The integral forms given in Eq. (9) are unsuitable for numerical computation, and consequently a more expeditious means for numerically evaluating $A_{m}, B_{m}$ will now be presented.

We first note that the Green's function of Eq. (6) can be written in the form [5]

$$
G\left(x, y ; x^{\prime}, y^{\prime}\right)=-\frac{1}{4 \pi \epsilon} \sum_{n=-\infty}^{\infty} \log \frac{\left(x-x^{\prime}\right)^{2}+\left(y-y^{\prime}-2 n\right)^{2}}{\left(x-x^{\prime}\right)^{2}+\left(y+y^{\prime}-2 n\right)^{2}} .
$$

The two terms of the summation corresponding to $n$ and $-n$, taken together, give the expression

$$
\begin{aligned}
\log \frac{\left[\left(x-x^{\prime}\right)^{2}+\left(y-y^{\prime}-2 n\right)^{2}\right]\left[\left(x-x^{\prime}\right)^{2}+\left(y-y^{\prime}+2 n\right)^{2}\right]}{\left[\left(x-x^{\prime}\right)^{2}+\left(y+y^{\prime}-2 n\right)^{2}\right]\left[\left(x-x^{\prime}\right)^{2}+\left(y+y^{\prime}+2 n\right)^{2}\right]} \\
=\log \frac{\left[1+\left(\frac{x-x^{\prime}+i\left(y-y^{\prime}\right)}{2 n}\right)^{2}\right]\left[1+\left(\frac{x-x^{\prime}-i\left(y-y^{\prime}\right)}{2 n}\right)^{2}\right]}{\left[1+\left(\frac{x-x^{\prime}+i\left(y+y^{\prime}\right)}{2 n}\right)^{2}\right]\left[1+\left(\frac{x-x^{\prime}-i\left(y+y^{\prime}\right)}{2 n}\right)^{2}\right]} \\
=\sum_{k=1}^{\infty} \frac{(-1)^{k} C_{k}\left(x, y ; x^{\prime}, y^{\prime}\right)}{k(2 n)^{2 k}}
\end{aligned}
$$

where

$$
\begin{aligned}
C_{k}\left(x, y ; x^{\prime}, y^{\prime}\right)= & {\left[\left(x-x^{\prime}\right)+i\left(y+y^{\prime}\right)\right]^{2 k}+\left[\left(x-x^{\prime}\right)-i\left(y+y^{\prime}\right)\right]^{2 k} } \\
& -\left[\left(x-x^{\prime}\right)+i\left(y-y^{\prime}\right)\right]^{2 k}-\left[\left(x-x^{\prime}\right)-i\left(y-y^{\prime}\right)\right]^{2 k} .
\end{aligned}
$$

Since $0<y, y^{\prime}<1$, a sufficient condition for the absolute convergence of the series is that $\left(x-x^{\prime}\right)^{2}<4\left(n^{2}-1\right)$. Thus for $\left(x-x^{\prime}\right)^{2}<140$ we have

$$
\begin{aligned}
G\left(x, y ; x^{\prime}, y^{\prime}\right)= & -\frac{1}{4 \pi \epsilon} \sum_{n=-5}^{5} \log \frac{\left(x-x^{\prime}\right)^{2}+\left(y-y^{\prime}-2 n\right)^{2}}{\left(x-x^{\prime}\right)^{2}+\left(y+y^{\prime}-2 n\right)^{2}} \\
& +\sum_{k=1}^{\infty} \alpha_{k} C_{k}\left(x, y ; x^{\prime}, y^{\prime}\right)
\end{aligned}
$$

where 


$$
\alpha_{k}=\frac{(-1)^{k}}{k 4^{k}} \sum_{n=6}^{\infty} n^{-2 k}
$$

can be computed from the Riemann Zeta Function or the Bernoulli numbers [7]. We may bound the $k$ th term of the remaining infinite series by

$$
\begin{aligned}
\left|\alpha_{k} C_{k}\left(x, y ; x^{\prime}, y^{\prime}\right)\right| & <4\left[\left(x-x^{\prime}\right)^{2}+4\right]^{k}\left|\alpha_{k}\right| \\
& =\frac{4}{k}\left\{\frac{\left(x-x^{\prime}\right)^{2}+4}{144}\right\}^{k}\left\{1+\sum_{n=7}^{\infty}\left(\frac{6}{n}\right)^{2 k}\right\} \\
& <\frac{26.2}{k}\left\{\frac{\left(x-x^{\prime}\right)^{2}+4}{144}\right\}^{k}
\end{aligned}
$$

so that for $\left|x-x^{\prime}\right| \leqq 3$ the use of terms through $k=10$ in Eq. (13) will give the Green's function to better than 10-place accuracy. We shall use this form of the Green's function to evaluate the integrals of Eq. (9).

Using [8] with a bit of reduction we obtain

$$
\begin{aligned}
\int_{0}^{2 \pi} \log \left[\left(x-x_{0}-R \cos \theta\right)^{2}+\left(y \pm y_{0} \pm R \sin \theta-2 n\right)^{2}\right] \cos m \theta d \theta \\
=\left\{\begin{array}{l}
2 \pi \log \left[\left(x-x_{0}\right)^{2}+\left(y \pm y_{0}-2 n\right)^{2}\right] \quad \text { for } m=0 \\
\pm 2 \pi \frac{R^{m}}{m} \frac{\cos \left\{m \operatorname{Arctan}\left[\left(y \pm y_{0}-2 n\right) /\left(x-x_{0}\right)\right]\right\}}{\left[\left(x-x_{0}\right)^{2}+\left(y \pm y_{0}-2 n\right)^{2}\right]^{m / 2}}
\end{array} \text { for } m=1,2,3, \cdots\right.
\end{aligned}
$$

and

$$
\begin{aligned}
& \int_{0}^{2 \pi} \log \left[\left(x-x_{0}-R \cos \theta\right)^{2}+\left(y \pm y_{0} \pm R \sin \theta-2 n\right)^{2}\right] \sin m \theta d \theta \\
& \quad=-2 \pi \frac{R^{m}}{m} \frac{\sin \left\{m \operatorname{Arctan}\left[\left(y \pm y_{0}-2 n\right) /\left(x-x_{0}\right)\right]\right\}}{\left[\left(x-x_{0}\right)^{2}+\left(y \pm y_{0}-2 n\right)^{2}\right]^{m / 2}} \text { for } m=1,2,3, \cdots .
\end{aligned}
$$

Since

$$
\begin{aligned}
C_{k}\left(x, y ; x_{0}\right. & \left.+R \cos \theta, y_{0}+R \sin \theta\right) \\
= & {\left[\left(x-x_{0}\right)+i\left(y+y_{0}\right)-R e^{-i \theta}\right]^{2 k}+\left[\left(x-x_{0}\right)-i\left(y+y_{0}\right)-R e^{i \theta}\right]^{2 k} } \\
& -\left[\left(x-x_{0}\right)+i\left(y-y_{0}\right)-R e^{i \theta}\right]^{2 k}-\left[\left(x-x_{0}\right)-i\left(y-y_{0}\right)-R e^{-i \theta}\right]^{2 k}
\end{aligned}
$$

we also have

$$
\begin{aligned}
\int C_{k}(x, y & \left.; x_{0}+R \cos \theta, y_{0}+R \sin \theta\right) e^{i m \theta} d \theta \\
& =2 \pi(-R)^{m}\left(\begin{array}{c}
2 k \\
m
\end{array}\right) \sum_{l=0}^{2 k-m} i^{l}\left(\begin{array}{c}
2 k-m \\
l
\end{array}\right)\left(x-x_{0}\right)^{2 k-m-l}\left\{\left(y_{0}+y\right)^{l}-\left(y_{0}-y\right)^{l}\right\}
\end{aligned}
$$

giving both the $\sin m \theta$ and $\cos m \theta$ integrals of $C_{k}$. Upon combining the above results, we obtain the final forms

$$
A_{0}\left(x, y ; x_{0}, y_{0}\right)=-\frac{1}{2 \epsilon} \log \frac{\cosh \pi\left(x-x_{0}\right)-\cos \pi\left(y-y_{0}\right)}{\cosh \pi\left(x-x_{0}\right)-\cos \pi\left(y+y_{0}\right)},
$$


(16)

$$
\begin{aligned}
A_{m}\left(x, y ; x_{0}, y_{0}\right)= & \frac{1}{2 m \epsilon} \sum_{n=-5}^{5}\left\{\frac{\cos m \operatorname{Arctan}\left[\left(y-y_{0}-2 n\right) /\left(x-x_{0}\right)\right]}{\left[\left(x-x_{0}\right)^{2}+\left(y-y_{0}-2 n\right)^{2}\right]^{m / 2}}\right. \\
\left.-\frac{\cos m \operatorname{Arctan}\left[\left(y+y_{0}-2 n\right) /\left(x-x_{0}\right)\right]}{\left[\left(x-x_{0}\right)^{2}+\left(y+y_{0}-2 n\right)^{2}\right]^{m / 2}}\right\} & \\
& -\frac{1}{2 \epsilon} \sum_{k=1}^{\infty} \alpha_{k}\left(\begin{array}{c}
2 k \\
m
\end{array}\right)_{l=0(2) 2 k-m}(-1)^{l / 2}\left(\begin{array}{c}
2 k-m \\
l
\end{array}\right) \\
& \times\left(x_{0}-x\right)^{2 k-m-l}\left\{\left(y_{0}+y\right)^{l}-\left(y_{0}-y\right)^{l}\right\} \\
B_{m}\left(x, y ; x_{0}, y_{0}\right)= & \frac{1}{2 m \epsilon} \sum_{n=-5}^{5}\left\{\frac{\sin m \operatorname{Arctan}\left[\left(y-y_{0}-2 n\right) /\left(x-x_{0}\right)\right]}{\left[\left(x-x_{0}\right)^{2}+\left(y-y_{0}-2 n\right)^{2}\right]^{m / 2}}\right. \\
& \left.+\frac{\sin m \operatorname{Arctan}\left[\left(y+y_{0}-2 n\right) /\left(x-x_{0}\right)\right]}{\left[\left(x-x_{0}\right)^{2}+\left(y+y_{0}-2 n\right)^{2}\right]^{m / 2}}\right\}, \\
& -\frac{1}{2 \epsilon} \sum_{k=1}^{\infty} \alpha_{k}\left(\begin{array}{c}
2 k \\
m
\end{array}\right) \sum_{l=1(2) 2 k-m}(-1)^{(l+1) / 2}(2 k-m) \\
& \times\left(x_{0}-x\right)^{2 k-m-l}\left\{\left(y_{0}+y\right)^{l}-\left(y_{0}-y\right)^{l}\right\}
\end{aligned}
$$

These expressions are well suited to numerical computation when $\left|x-x_{0}\right| \leqq 3$ in which case the infinite series remaining in Eqs. (16)-(17) are rapidly convergent.

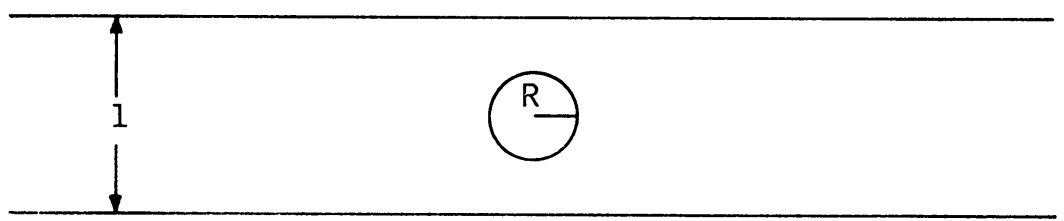

Figure 2. Capacitance for a Circular Conductor Centered between Parallel Ground Lines.

\begin{tabular}{rrr}
$R$ & \multicolumn{1}{c}{$C / \epsilon$} & $M$ \\
\hline .05 & 2.46967 & 1 \\
.10 & 3.39473 & 2 \\
.15 & 4.34868 & 2 \\
.20 & 5.43684 & 2 \\
.25 & 6.76127 & 4 \\
.30 & 8.48134 & 6 \\
.35 & 10.91395 & 6 \\
.40 & 14.86438 & 8 \\
.45 & 23.49826 & 12 \\
\hline
\end{tabular}

Note. $\epsilon$ is the permittivity constant for the medium between the ground lines. For free space $\epsilon=8.8541710^{-12} \mathrm{farad} /$ meter $(M K S)$ or $1 / 4 \pi$ (Gaussian).

5. Cost of Computation. The calculational procedure which has been described provides a relatively inexpensive alternative to the finite-difference methods which 
may also be used to solve boundary value problems of the type considered. Almost all of the computational time required to calculate the $N^{2}$ elements of the capacitance matrix $\left\{C_{i j}\right\}$ is spent evaluating the $4 N^{2} M(2 M+1)$ elements $A_{m}\left(X_{i k}, Y_{i k} ; x_{j}, y_{j}\right)$, $B_{m}\left(X_{i k}, Y_{i k} ; x_{j}, y_{j}\right)$ which are needed for the analysis. (It will be noted that these arrays are independent of the boundary conditions of Eq. (3) so that once these arrays have been computed the solution for an arbitrary choice of $V_{i}$ may be obtained by solving an $N^{2}(2 M+1)$ th order system of linear equations.) Thus for a fixed $M$ we see that the computational time is roughly proportional to $N^{2}$. From

$$
\begin{aligned}
& \overline{1} \\
& C_{11} / \epsilon=3.39473 \\
& \mathrm{C}_{11} / \epsilon=\mathrm{C}_{22^{/ \epsilon}}=3.88153 \\
& C_{12} / \epsilon=C_{21} / \epsilon=-1.26037 \\
& 123 \\
& 000 \\
& \mathrm{C}_{11} / \epsilon=\mathrm{C}_{33} / \epsilon=3.88184 \\
& \mathrm{C}_{22} / \epsilon=4.35963 \\
& C_{12} / \epsilon=C_{21} / \epsilon=C_{23} / \epsilon=C_{32} / \epsilon=-1.24832 \\
& C_{13} / \epsilon=C_{31} / \epsilon=-.03371 \\
& \begin{array}{llll}
1 & 2 & 3 & 4 \\
0 & 0 & 0 & 0
\end{array} \\
& 12 \\
& 00 \\
& \begin{array}{l}
\mathrm{C}_{11} / \epsilon=\mathrm{C}_{44} / \epsilon=3.88184 \\
\mathrm{C}_{22} / \epsilon=\mathrm{C}_{33} / \epsilon=4.35993
\end{array} \\
& C_{12} / \epsilon=C_{21} / \epsilon=C_{34} / \epsilon=C_{43} / \epsilon=-1.24830 \\
& \mathrm{C}_{23} / \epsilon=\mathrm{C}_{32} / \epsilon=-1.23651 \\
& C_{13} / \epsilon=C_{31} / \epsilon=C_{24} / \epsilon=C_{42} / \epsilon=-.03304 \\
& c_{14} / \epsilon=C_{41} / \epsilon=-.00188 \\
& \mathrm{C}_{11} / \epsilon=\mathrm{C}_{55^{/ \epsilon}}=3.88184 \\
& \mathrm{C}_{22} / \epsilon=\mathrm{C}_{44} / \epsilon=4.35993 \\
& \mathrm{C}_{33} / \mathrm{\epsilon}=4.36023 \\
& \mathrm{C}_{12} / \mathbf{\epsilon}=\mathrm{C}_{21} / \mathbf{\epsilon}=\mathrm{C}_{45} / \mathbf{\epsilon}=\mathrm{C}_{54} / \mathbf{\epsilon}=-1.24830 \\
& \mathrm{C}_{23} / \epsilon=\mathrm{C}_{32} / \epsilon=\mathrm{C}_{34} / \epsilon=\mathrm{C}_{43} / \epsilon=-1.23649 \\
& C_{13} / \epsilon=C_{31} / \epsilon=C_{35} / \epsilon=C_{53} / \epsilon=-.03303 \\
& \mathrm{C}_{24} / \epsilon=\mathrm{C}_{42} / \epsilon=-.03238 \\
& C_{14} / \epsilon=C_{41} / \epsilon=C_{25} / \epsilon=C_{52} / \epsilon=-.00185 \\
& \mathrm{C}_{15} / \epsilon=\mathrm{C}_{51} / \epsilon=-.00011
\end{aligned}
$$

\begin{tabular}{lllll}
1 & 2 & 3 & 4 & 5 \\
0 & 0 & 0 & 0 & 0 \\
\hline
\end{tabular}

Figure 3. Capacitance Matrices for Systems of 1, 2, 3, 4, and 5 Circular Conductors

Note. The separation of the ground lines is 1. , the radius of each conductor is $R=.1$, and the center of the $i$ th conductor is given by $\left(x_{i}, y_{i}\right)=(.4 i, .5), i=1$, $\cdots, 5$. 
experience with the technique, we might note that the use of $M=6$ will give 6 place approximations to the capacitances $C_{i j}$ (assuming that the conductors are separated by a distance of at least .1), and in this case the cost of the computation is about $15 \mathrm{~N}^{2}$ seconds on the IBM 7044 computer.

6. Sample Results. The calculation of the capacitance of a single circular conductor of radius $R$ with center at $(0, .5)$ has been undertaken using the above numerical process. Figure 2 presents the capacitance as a function of $R$ for selected values of $R$ along with the value of $M$ needed to achieve convergence to within $\pm 5 \cdot 10^{-6}$. These results when rounded to 4 significant figures agree precisely with those published by Knight [1]. The capacitance matrices for systems of 1, 2, 3, 4, and 5 equally spaced centered conductors having a common radius $R=.1$ and center-to-center spacing $d=.4$ are presented in Figure 3.

Acknowledgement. This work was supported by the U. S. Naval Weapons Center, China Lake, California under Contract N00123-67-C-0714.

Research and Development Laboratory, Equipment Group

Texas Instruments, Inc.

Dallas, Texas 75222

1. R. C. KNIGHT, "The potential of a circular cylinder between two infinite planes," Proc. London Math. Soc., v. 39, 1935, pp. 272-281.

2. J. W. CraGGS, "The determination of capacity for two-dimensional systems of cylindrical conductors," Quart. J. Math. Oxford Ser., v. 17, 1946, pp. 131-137. MR 8, 298.

3. E. G. CRISTAL, "Coupled circular cylindrical rods between parallel grounded planes," IEEE Trans., v. MTT-12, 1964, pp. 422-439.

4. O. D. Kellogg, Foundations of Potential Theory, Dover, New York, 1953. p. 159

5. L. V. Bewley, Two Dimensional Fields in Electrical Engineering, Dover, New York, 1963,

6. J. C. Maxwell, A Treatise on Electricity and Magnetism. Vol. 1, 3rd ed., Dover, New York, 1954, pp. 103-118. MR 16, 99.

7. M. Abramowitz \& I. A. Stegun (Editors), Handbook of Mathematical Functions, with Formulas, Graphs and Mathematical Tables, Nat. Bur. Standards, Appl. Math. Series, 55, Superintendent of Documents, U. S. Government Printing Office, Washington, D. C., 1964, pp. 807, 811; 3rd printing with corrections, 1965. MR 29 \#4914; MR 31 \#1400.

8. H. B. Dwighr, Tables of Integrals and Other Mathematical Data, 4th ed., Macmillan, New York, 1961, MR 23 \#B2613. 